\title{
Logarithmic forms with twisted coefficients
}

\author{
Philip A. Foth ${ }^{1}$
}

March 3, 2022

\begin{abstract}
Given a compact Kähler manifold, we consider the complement $U$ of a divisor with normal crossings and a unitary local system $\tilde{\mathfrak{g}}$ on it. We consider a differential graded Lie algebra of forms with holomorphic logarithmic singularities and vanishing residues. We construct a spectral sequence converging to the $L_{2}$ cohomology $H^{\bullet}(U, \tilde{\mathfrak{g}})_{(2)}$ corresponding to the anti-holomorphic filtration of this algebra and compute its $E_{1}$ term. This differential graded Lie algebra gives an example of a formal algebra which does not obey the $d d^{c}$-Lemma.
\end{abstract}

\section{Introduction}

Let $X$ be a compact Kähler manifold and let $D \subset X$ be a divisor with normal crossings on $X$ which has smooth irreducible components. We let $U$ be the complement: $U=X \backslash D$, and $j: U \hookrightarrow X$ be the inclusion. The Hironaka resolution of singularities theorem [6] asserts that every smooth quasi-projective variety is birational to a manifold $U$ of this type. Let $\tilde{\mathfrak{g}}$ be a unitary local system on $U$, let $V$ be the corresponding holomorphic vector bundle over $U$, and let $\bar{V}$ be its canonical holomorphic extension [1].

In this paper we construct a differential graded Lie algebra (DGLA) of smooth differential forms on $U$ with coefficients in $\bar{V}$ which have logarithmic singularities along $D$ and vanishing residues. This algebra admits the following simple local description for a local system of rank 1 . Let $x \in X$ and let

\footnotetext{
${ }^{1}$ Sloan Doctoral Dissertation Fellow
} 
$\Delta$ be a polydisc around $x$ such that in local holomorphic coordinate system $\Delta \cap D$ is given by $z_{1} \cdots z_{l}=0$. Then over $\Delta$ the sections of the sheaf $\tilde{A}_{X}^{q}(\bar{V})$ are generated by $\left(A_{X}^{q} \otimes \bar{V}\right)_{\mid \Delta}$ and by all expressions of the form:

$$
\frac{d z_{j_{1}}}{z_{j_{1}}} \wedge \cdots \wedge \frac{d z_{j_{k}}}{z_{j_{k}}} \wedge \tau \otimes \nu,
$$

where $\tau \in A_{X}^{q-k}, \nu \in \Gamma(\Delta, \bar{V}), k \leq q, 1 \leq j_{1}<\cdots<j_{k} \leq l$ and such that the monodromy around $D_{j_{s}}:=\left\{z_{j_{s}}=0\right\}$ for all $1 \leq s \leq k$ is nontrivial. In the holomorphic context this algebra already appeared in a work of Timmerscheidt [7]. We say that a DGLA is formal if it is quasi-isomorphic to its cohomology algebra.

THEOREM 2.3 The $d^{\prime}$-cohomology of the double complex $\tilde{A}_{X}^{p, q}(\bar{V})$ is equal to

$$
E_{1}^{p, q}=H^{p}\left(X, \bar{\Omega}^{q} \otimes j_{*} \tilde{\mathfrak{g}}\right),
$$

where $\bar{\Omega}^{q}$ is the sheaf of the anti-holomorphic q-forms on $X$.

We notice that in general the groups $H^{p}\left(X, \bar{\Omega}^{q} \otimes j_{*} \tilde{\mathfrak{g}}\right)$ are infinite dimensional.

One of the important ingredients in the course of our proof is an analogue of the $\bar{\partial}$-Poincaré lemma. The DGLA $\tilde{A}_{X}^{\bullet}(\bar{V})$ is naturally bigraded by holomorphic and anti-holomorphic degrees. Our Lemma 2.2 tells us that locally the complex

$$
\ldots \stackrel{\nabla^{\prime}}{\rightarrow} \tilde{A}_{X}^{p-1, q}(\bar{V}) \stackrel{\nabla^{\prime}}{\rightarrow} \tilde{A}_{X}^{p, q}(\bar{V}) \stackrel{\nabla^{\prime}}{\rightarrow} \tilde{A}_{X}^{p+1, q}(\bar{V}) \stackrel{\nabla^{\prime}}{\rightarrow} \ldots
$$

is exact.

One of the statements that we obtain (essentially using results in [7]) is that the corresponding spectral sequence with

$$
E_{1}^{p, q}=H^{p}\left(X, \bar{\Omega}^{q} \otimes j_{*} \tilde{\mathfrak{g}}\right)
$$

abuts to $H^{p, q}(U, \tilde{\mathfrak{g}})_{(2)}$. Another important conclusion is that the $d^{\prime} d^{\prime \prime}$-Lemma does not hold for our DGLA $\tilde{A}_{X}^{\bullet}(\bar{V})$. Nevertheless, the DGLA $\tilde{A}_{X}^{\bullet}(\bar{V})$ is formal, because it is a sub-DGLA of another formal DGLA $B^{\bullet}(\tilde{\mathfrak{g}})$ and has the same cohomology. We refer to the author's paper [4] for the construction of $B^{\bullet}(\tilde{\mathfrak{g}})$ and a proof of its formality. 
Acknowledgments. I am tremendously indebted to Jean-Luc Brylinski for his advice and care.

\section{Forms with logarithmic poles}

Let $X$ be a compact Kähler manifold of complex dimension $d$ endowed with a Kähler form $\lambda$ and let $D$ be a divisor on $X$ with normal crossings which can be represented as a union of $r$ smooth irreducible complex-analytic subvarieties of codimension 1:

$$
D=\bigcup_{i=1}^{r} D_{i}
$$

We let $G=U(N)$ and $\mathfrak{g}=\mathfrak{u}(N)$. We let $U:=X \backslash D$, we let the map $j: U \hookrightarrow X$ be the inclusion.

A differential graded Lie algebra $(L, d)$ consists of a graded Lie algebra

$$
L=\oplus_{i \geq 0} L^{i}, \quad[,]: L^{i} \times L^{j} \rightarrow L^{i+j},
$$

satisfying for $\alpha \in L^{i}, \beta \in L^{j}$, and $\gamma \in L^{k}$ :

$$
\begin{gathered}
{[\alpha, \beta]+(-1)^{i j}[\beta, \alpha]=0} \\
(-1)^{k i}[\alpha,[\beta, \gamma]]+(-1)^{i j}[\beta,[\gamma, \alpha]]+(-1)^{j k}[\gamma,[\alpha, \beta]]=0
\end{gathered}
$$

together with a derivation $d$ of degree 1 (also called a differential):

$$
d: L^{i} \rightarrow L^{i+1}, d \cdot d=0, d[\alpha, \beta]=[d \alpha, \beta]+(-1)^{i}[\alpha, d \beta] .
$$

The main examples of DGLA we will deal with include $\left(\tilde{A}_{X}^{i}(\bar{V}), d^{\prime}+d^{\prime \prime}\right)$, where

$$
\tilde{A}_{X}^{i}(\bar{V}):=\bigoplus_{p+q=i} \tilde{A}_{X}^{p, q}(\bar{V}) .
$$

The cohomology of any DGLA is a DGLA too, considered with zero differential. We say that two DGLAs $\left(L_{1}, d_{1}\right)$ and $\left(L_{2}, d_{2}\right)$ are quasi-isomorphic if there exists a third DGLA $\left(L_{3}, d_{3}\right)$ and DGLA homomorphisms $i$ and $p$ :

$$
\left(L_{1}, d_{1}\right) \stackrel{i}{\leftarrow}\left(L_{3}, d_{3}\right) \stackrel{p}{\rightarrow}\left(L_{2}, d_{2}\right)
$$


such that both $i$ and $p$ induce isomorphisms in cohomology. It turns out that on the set of DGLAs quasi-isomorphism is an equivalence relation. We also say that a DGLA is formal if it is quasi-isomorphic to its cohomology [3].

In order to construct our DGLA we introduce more notation: let $D^{(m)}$ be the subvariety consisting of points which belong to at least $m$ irreducible components of the divisor $D$, e. g. $D^{(0)}=X$ and $D^{(1)}=D$. Let $\tilde{D}^{(m)} \rightarrow$ $D^{(m)}$ be normalization maps and let

$$
v^{m}: \tilde{D}^{(m)} \rightarrow D^{(m)} \hookrightarrow X
$$

be th composition of normalizations with inclusions. We also let $\tilde{C}^{(m)}:=$ $\left(v^{m}\right)^{-1}\left(D^{(m+1)}\right)$ which is a divisor with normal crossings on $\tilde{D}^{(m)}$ (possibly empty).

Given a unitary local system $\tilde{\mathfrak{g}}$ on $U$, let $(\bar{V}, \nabla)$ be the Deligne extension of $V$. Here $\bar{V}$ is a holomorphic vector bundle over $X$ and $\nabla$ is a holomorphic connection with logarithmic singularities along $D$. We refer the reader to [1] for the details. The connections are extended as usual to holomorphic forms with logarithmic poles.

There exist [2] higher residue maps $\operatorname{Res}_{m}(\bar{V})$ which are morphisms of complexes:

$$
\operatorname{Res}_{m}(\bar{V}): \quad \Omega_{X}^{\bullet}\langle D\rangle \otimes \bar{V} \rightarrow v_{*}^{m}\left(\Omega_{\tilde{D}^{(m)}}^{\bullet}\left\langle\tilde{C}^{(m)}\right\rangle \otimes \bar{V}_{m}\right)[-m] .
$$

Here $\bar{V}_{m}$ is the unique vector subbundle of $\left(v^{m}\right)^{*} \bar{V}$ equipped with a unique holomorphic integrable connection $\nabla_{m}$ with logarithmic poles along $\tilde{C}^{(m)}$ such that

$$
\left.\operatorname{Ker} \nabla_{m}\right|_{\left[\tilde{D}^{(m)} \backslash \tilde{C}^{(m)}\right]}=\left(v_{m}\right)^{-1}\left(\left.\left(j_{*} \tilde{\mathfrak{g}}\right)\right|_{\left[\tilde{D}^{(m)} \backslash \tilde{D}^{(m+1)}\right]}\right) .
$$

This means that

$$
\left(\bar{V}_{m}, \nabla_{m}\right)
$$

is the canonical extension of

$$
\left(v_{m}\right)^{-1}\left(\left.\left(j_{*} \tilde{\mathfrak{g}}\right)\right|_{\left[\tilde{D}^{(m)} \backslash \tilde{D}^{(m+1)}\right]}\right) .
$$

As usual, we denote by $\Omega_{X}^{\bullet}\langle D\rangle$ the complex of sheaves of holomorphic forms on $U$ with logarithmic poles along $D$. The fact that $\operatorname{Res}_{m}(\bar{V})$ is a morphism of complexes means that

$$
\operatorname{Res}_{m}(\bar{V}) \circ \nabla=\nabla_{m} \circ \operatorname{Res}_{m}(\bar{V}) .
$$


Let us define

$$
\tilde{\Omega}_{X}^{\bullet}(\bar{V}):=\operatorname{Ker} \operatorname{Res}_{1}(\bar{V}) ;
$$

it is a complex of sheaves comprised of holomorphic forms on $X \backslash D$ with coefficients in $\bar{V}$ which have logarithmic poles along $D$ and have no residues. The residue maps take values in the part of the local system invariant under all the monodromy transformations. So when all the eigenvalues of the monodromy transformations are different from 1 , the complexes $\tilde{\Omega}_{X}^{\bullet}(\bar{V})$ and $\Omega_{X}^{\bullet}\langle D\rangle \otimes \bar{V}$ coincide.

The complex $\tilde{\Omega}_{X}^{\bullet}(\bar{V})$ admits a different description [7]. First, on $U$ we let $\tilde{\Omega}_{X}^{p}(\bar{V})_{\mid U}:=\Omega_{U}^{p} \otimes \bar{V}_{\mid U}$. For $x \in D$ we pick a small polycylinder $\Delta$ containing $x$ such that $D \cap \Delta$ is given by $z_{1} \cdots z_{l}=0$ in local holomorphic coordinates $z_{1}, \ldots, z_{d}$. Let $T_{i}, 1 \leq i \leq l$, be the monodromy transformation of $\tilde{\mathfrak{g}}_{\mid \Delta \backslash D}$ around $D_{i}$. Let $\tilde{\Omega}_{X}^{1}(\bar{V})_{\mid \Delta} \subset \Omega_{X}^{1}\langle D\rangle \otimes \bar{V}_{\mid \Delta}$ be generated over $\mathcal{O}_{\Delta}$ by $\Omega_{\Delta}^{1} \otimes_{\mathcal{O}_{\Delta}} \bar{V}_{\mid \Delta}$ and

$$
\Omega_{\Delta}^{1}\left\langle D_{i}\right\rangle \otimes_{\mathbb{C}}\left(\operatorname{Ker}\left(T_{i}-I d\right)\right)^{\perp},
$$

where $\left(\operatorname{Ker}\left(T_{i}-I d\right)\right)^{\perp}$ is the orthogonal complement of the local subsystem $\operatorname{Ker}\left(T_{i}-I d\right)$ of $\tilde{\mathfrak{g}}_{\Delta \backslash D}$, and $1 \leq i \leq l$. Similarly we define the groups $\tilde{\Omega}_{X}^{p}(\bar{V})_{\mid \Delta}$. Those sheaves glue together nicely to a subcomplex $\left(\tilde{\Omega}_{X}^{\bullet}(\bar{V}), \nabla\right)$ of the complex of sheaves of meromorphic differential forms on $X$ with coefficients in $\bar{V}$ with logarithmic poles along $D$.

Let us introduce a double complex

$$
\tilde{A}_{X}^{p, q}(\bar{V}):=\Gamma\left(X, \tilde{\Omega}_{X}^{p}(\bar{V}) \otimes_{\mathcal{O}_{X}} \underline{A}_{X}^{0, q}\right),
$$

where $\underline{A}_{X}^{0, q}$ is the sheaf of $C^{\infty}$ differential forms on $X$ of type $(0, q)$. We need to establish certain results analogous, in a sense, to the $d^{\prime}$-Poincaré Lemma.

LEMMA 2.1 Let $\kappa \in \mathbb{R}, 0<\kappa<1$. Given a complex $C^{\infty}$ function $f$ in an open neighbourhood $W_{1}$ of the closure $\bar{\Delta}$ of a disk $\Delta \subset \mathbb{C}$ centered at zero, there exists a $C^{\infty}$ function $g$ in an open neighbourhood $W_{2} \subset W_{1}$ of $\bar{\Delta}$ such that

$$
z \frac{\partial g}{\partial z}+\kappa g=f
$$

in $W_{2}$. Moreover, if $f$ is $C^{\infty}$ or holomorphic in some additional parameters, then $g$ can be chosen to have the same properties. 
Proof. Denote

$$
P=z \frac{\partial}{\partial z}+\kappa
$$

One of the indications that the lemma is true is that on monomials of the form $z^{a} \bar{z}^{b}$ the operator $P$ is bijective, since

$$
P \frac{z^{a} \bar{z}^{b}}{a+\kappa}=z^{a} \bar{z}^{b}
$$

Also, since the closure $\bar{\Delta}$ is compact, we may and will assume that $f$ vanishes outside a compact subset of $\mathbb{C}$.

Next, we decompose

$$
f(z)=\sum_{n \in \mathbb{Z}} f_{n}(z)
$$

where

$$
f_{n}(z)=\frac{1}{2 \pi} \int_{0}^{2 \pi} f\left(e^{\sqrt{-1} \phi} z\right) e^{-\sqrt{-1} n \phi} d \phi
$$

and, clearly, we have

$$
f_{n}\left(e^{\sqrt{-1} \phi} z\right)=e^{\sqrt{-1} n \phi} f_{n}(z)
$$

This series is well known to be locally uniformly convergent together with all partial derivatives. Now we claim that when $n \geq 0$ :

$$
f_{n}(z)=e^{\sqrt{-1} n \phi} r^{n} h_{n}\left(r^{2}\right)
$$

and when $n \leq 0$ :

$$
f_{n}(z)=e^{\sqrt{-1} n \phi} r^{-n} h_{n}\left(r^{2}\right),
$$

where $r, \phi$ are the standard polar coordinates, $r \geq 0, z=r e^{\sqrt{-1} \phi}$, and $h_{n}\left(r^{2}\right)$ is a smooth $C^{\infty}$ function. First, it is clear that $f_{n}(z)=e^{\sqrt{-1} n \phi} s_{n}(r)$, where $s_{n}(r)$ is a smooth function.

Let us represent for small $r$

$$
f_{n}(z)=\sum_{i=\max (0, n)}^{k} a_{i} z^{i} \bar{z}^{i-n}+o\left(r^{k}\right) .
$$

Further we have

$$
f_{n}(z)=e^{\sqrt{-1} n \phi}\left[\sum_{i=\max (0, n)}^{k} a_{i} r^{2 i-n}+o\left(r^{k}\right)\right] .
$$


So we have

$$
h_{n}(r)=\sum_{i=\max (0, n)}^{k} a_{i} r^{2 i-n-|n|}+o\left(r^{k-|n|}\right) .
$$

The first term has $r$ to the even power and the second term has derivatives with respect to $r^{2}$ up to the order $(k-|n|) / 2$. Thus $h_{n}$ is a smooth $C^{\infty}$ function in $r^{2}$, because all its (one-sided) derivatives $d h_{n} / d\left(r^{2}\right)$ of all orders do exist at $r=0$.

Let us now try to solve the equation $P g_{n}=f_{n}$. First, we do it for $n \geq 0$. We use the coordinates $\left(a=r^{2}, \phi\right)$, define $\partial / \partial a$ and $\partial / \partial \phi$ accordingly and notice that

$$
z \frac{\partial}{\partial z}=a \frac{\partial}{\partial a}+\frac{1}{2 \sqrt{-1}} \frac{\partial}{\partial \phi} .
$$

We will look for a solution $g_{n}(z)$ also in the form $g_{n}(z)=e^{\sqrt{-1} n} \phi r^{n} w_{n}\left(r^{2}\right)$. Applying $p$ to $g_{n}(z)$ one gets

$$
P g_{n}(z)=\left((n+\kappa) w_{n}(a)+a \frac{\partial w_{n}}{\partial a}\right) e^{\sqrt{-1} n \phi} r^{n} .
$$

Comparing $P g_{n}$ and $f_{n}$ we see that we need to solve the equation

$$
a \frac{\partial w_{n}(a)}{\partial a}+(n+\kappa) w_{n}(a)=h_{n}(a)
$$

on an interval $[0, \varepsilon)$. The solution is given by

$$
w_{n}(a)=\frac{1}{a^{n+\kappa}} \int_{0}^{a} b^{n+\kappa-1} h_{n}(b) d b .
$$

It is clear right from the formula that the solution $w_{n}(a)$ is smooth.

Now, we solve $P g_{n}=f_{n}$ for $n \leq 0$. Here the situation is a bit simpler and we have the equation

$$
a \frac{\partial w_{n}(a)}{\partial a}+\kappa w_{n}(a)=h_{n}(a)
$$

on an interval $[0, \varepsilon)$, which is the same as the case $n=0$ considered above.

The last thing for us to do in this Lemma is to show that the series $\sum g_{n}(z)$ converges uniformly to a smooth $C^{\infty}$ function on compact subsets. 
Explicitly, for $n \geq 0$ (as we have noticed the case $n \leq 0$ is the same as $n=0$ ) we have:

$$
\left|\frac{1}{a^{n+\kappa}} \int_{0}^{a} b^{n+\kappa-1} h_{n}(b) d b\right| \leq \frac{\sup _{b \in[0, a]}\left|h_{n}(b)\right|}{|n+\kappa-1|} .
$$

Now we have

$$
\sum_{n \geq 0}\left|g_{n}(z)\right| \leq \sum_{n \geq 0} r^{n}\left|w_{n}\left(r^{2}\right)\right| \leq \sum_{n \geq 0} r^{n} \frac{\sup _{b \in[0, a]}\left|h_{n}(b)\right|}{|n+\kappa-1|}<+\infty
$$

for small $r$. For the first derivative we use integration by parts:

$$
\begin{gathered}
\frac{d}{d a}\left(\frac{1}{a^{n+\kappa}} \int_{0}^{a} b^{n+\kappa-1} h_{n}(b) d b\right)= \\
=\frac{1}{a^{n+\kappa}} a^{n+\kappa-1} h_{n}(a)-(n+\kappa) \frac{1}{a^{n+\kappa+1}} \int_{0}^{a} b^{n+\kappa-1} h_{n}(b) d b= \\
=\frac{1}{a} h_{n}(a)-\frac{n+\kappa}{a^{n+\kappa+1}}\left\{\left[\frac{-1}{n+\kappa} b^{n+\kappa} h_{n}(b)\right]_{0}^{a}+\frac{1}{n+\kappa} \int_{0}^{a} b^{n+\kappa} \frac{d h_{n}}{d b} d b\right\}= \\
=-\frac{1}{a^{n+\kappa+1}} \int_{0}^{a} b^{n+\kappa} \frac{d h_{n}}{d b} d b .
\end{gathered}
$$

This is again bounded in norm by

$$
\frac{\sup _{b \in[0, a]}\left|\frac{d h_{n}(b)}{d b}\right|}{|n+\kappa|} .
$$

Therefore, the absolute sum of the first derivatives converges as well. Clearly, the same arguments work for all the derivatives.

We denote by $d^{\prime}$ the component of type $(1,0)$ of the connection $\nabla$.

LEMMA 2.2 For $p>0, x \in X$ let $\omega$ be an element of $\tilde{A}_{\Delta}^{p, q}(\bar{V})$ defined in an open neighbourhood of a polydisc $\bar{\Delta}$ containing $x$ such that $d^{\prime} \omega=0$. Then there exists $\alpha \in \tilde{A}_{\Delta}^{p-1, q}(\bar{V})$ defined in an open neighbourhood of $\bar{\Delta}$ such that $\omega=d^{\prime} \alpha$.

Proof. We can easily reduce to the case when $q=0$, so henceforth we assume that all the forms we deal with belong to $\tilde{A}_{\Delta}^{\bullet, 0}(\bar{V})$. 
The statement is clear when $x \in U$. Locally over $\Delta \backslash D$ our local system $\tilde{\mathfrak{g}}$ decomposes into a direct sum of local systems of rank one (since $\pi_{1}(\Delta \backslash D) \simeq$ $\mathbb{Z}^{d}$ is commutative). Therefore we can assume that we have a unitary local system of rank 1 . Let $x \in D$ and first, let us work with the case $d=1$ when the monodromy around $z=0$ is equal to $e^{-\sqrt{-1} \theta}$, where $0<\theta<2 \pi$. Thus if we have a multi-valued horizontal section $s: \nabla s=0$ then the monodromy operator $T$ acts on it as $T s=e^{-\sqrt{-1} \theta} s$. Let us consider the uni-valued section $\mu=z^{\theta / 2 \pi} s$ which spans the canonical extension. We have:

$$
\nabla \mu=\frac{\theta}{2 \pi} \frac{d z}{z} \otimes \mu
$$

and the Leibnitz rule also imply that for any smooth function $f(z) \in C^{\infty}(\Delta)$, we have

$$
\nabla(f \mu)=\left[d^{\prime} f+\frac{\theta}{2 \pi} f \frac{d z}{z}\right] \otimes \mu=\left(z \frac{\partial f}{\partial z}+\frac{\theta}{2 \pi} f\right) \frac{d z}{z} \otimes \mu .
$$

Letting $\kappa:=\theta / 2 \pi$ we are within the scope of Lemma 2.1 and thus we are done in this case.

Next, we shall apply standard arguments [5] to treat the case when $x \in$ $D^{(d)}$ and $\Delta \cap D$ is given by $z_{1} z_{2} \cdots z_{d}=0$ and the monodromy around $D_{i}$ (corresponding to $z_{i}=0$ ) is $e^{-\sqrt{-1} \theta_{i}}, 0<\theta_{i}<2 \pi$. We denote by $P_{i}$ the operator $z_{i} \frac{\partial}{\partial z_{i}}+\frac{\theta_{i}}{2 \pi}$ for $1 \leq i \leq d$.

Let $\omega=\frac{d z_{k}}{z_{k}} \wedge \psi+\beta$ where $\psi$ and $\beta$ do not involve $\frac{d z_{j}}{z_{j}}$ for $j \geq k$ and we will do the proof by induction on $k$. For $k=0$ the statement is obvious. Since $0=d^{\prime} \omega=-\frac{d z_{k}}{z_{k}} \wedge d^{\prime}(\psi)+d^{\prime}(\beta)$, it is clear that all the separate coefficients of the forms $\psi$ and $\beta$ must be killed by the operators $P_{j}$ for $j>k$.

Any coefficient $f$ in the explicit representation of the differential form $\psi$ (with coefficients in $\bar{V}$ ) can be viewed as a $C^{\infty}$ function of the single variable $z_{k}$ in an open neighbourhood of the disk $\bar{\Delta}_{1} \subset \mathbb{C}$ obtained as the projection of $\Delta$ on the $k$-th coordinate. The function $f$ is also a $C^{\infty}$ function of the auxiliary parameters $z_{1}, \ldots, z_{k-1}$ in the polydisk $\bar{\Delta}_{k-1} \subset \mathbb{C}^{k-1}$ obtained by the projection of $\Delta$ onto the first $(k-1)$ coordinates. It follows from Lemma 2.1 that there exists a $C^{\infty}$ function $g$ in an open neighbourhood of $\bar{\Delta}$ such that $P_{k} g=f$ and that $g$ retains all the properties of $f$ with respect to other coordinates. Let $\Omega$ be the differential form obtained from $\psi$ by replacing each coefficient $f$ by the corresponding coefficient $g$. It follows that

$$
d^{\prime} \Omega=\delta+\frac{d z_{k}}{z_{k}} \wedge \psi
$$


where $\delta$ is a differential form which does not involve $\frac{d z_{j}}{z_{j}}$ for $j \geq k$. Next we let $u=\omega-d^{\prime} \Omega=\beta-\delta$ and we notice that $u$ does not involve $\frac{d z_{j}}{z_{j}}$ for $j \geq k$, since $\beta$ and $\delta$ are such. Besides,

$$
d^{\prime} u=d^{\prime} \omega-d^{\prime} d^{\prime} \Omega=0 .
$$

By the induction hypothesis there exists a $\left(C^{\infty}\right)$ differential form $w$ in an open neighbourhood of $\bar{\Delta}$ such that $u=d^{\prime} w$. If we let now $\alpha=w+\Omega$ then $d^{\prime} \alpha=\omega$.

In the general case we can ignore the components of the divisor $D$ which correspond to the trivial monodromy and by separating variables and applying the standard $d^{\prime}$-Lemma the above arguments will still work when $\Delta \cap D$ is given locally by $z_{1} \cdots z_{l}=0$ and $0 \leq l \leq d$.

Let us notice that another way to prove the above Lemma is to consider the Koszul complex

$$
\cdots \stackrel{\mathcal{P}}{\rightarrow} E^{j} \stackrel{\mathcal{P}}{\rightarrow} E^{j+1} \stackrel{\mathcal{P}}{\rightarrow} \cdots,
$$

where $\mathcal{P}$ is the family of commutative operators $\left(P_{1}, \ldots, P_{l}\right)$ and

$$
E^{j}:=\bigoplus_{|I|=j} \mathfrak{A} \otimes \frac{d z_{I}}{z_{I}}
$$

where $\mathfrak{A}$ is the algebra of germs of $C^{\infty}$ functions at zero. Since Lemma 2.1 tells us that $P_{j}$ is invertible, the complex is exact. Let us denote by $\bar{\Omega}^{q}$ the sheaf of anti-holomorphic $q$-forms.

THEOREM 2.3 The $d^{\prime}$-cohomology of the double complex $\tilde{A}_{X}^{p, q}(\bar{V})$ is equal to

$$
E_{1}^{p, q}=H^{p}\left(X, \bar{\Omega}^{q} \otimes j_{*} \tilde{\mathfrak{g}}\right)
$$

Proof. The local exactness of the rows of the initial sheet of the spectral sequence $E_{0}^{p, q}=\tilde{A}_{X}^{p, q}(\bar{V})$ with the differential $d^{\prime}$ allows us to identify

$$
E_{1}^{p, q}=H^{p}\left(X, \operatorname{Ker}\left[d^{\prime}: \tilde{A}_{X}^{0, q}(\bar{V}) \rightarrow \tilde{A}_{X}^{1, q}(\bar{V})\right]\right) .
$$


Next, we notice that the operator

$$
P=z \frac{\partial}{\partial z}+\kappa
$$

has no kernel in $C^{\infty}$ functions on $\mathbb{C}$ when, $0<\kappa<1$ and the kernel consists of exactly the anti-holomorphic functions when $\kappa=0$. Therefore, for a local system of rank one, the space $\operatorname{Ker}\left[d^{\prime}: \tilde{A}_{X}^{0, q}(\bar{V}) \rightarrow \tilde{A}_{X}^{1, q}(\bar{V})\right]$ consists of the anti-holomorphic forms with support not including the components of the divisor with non-trivial monodromy. This shows in general, that

$$
\operatorname{Ker}\left[d^{\prime}: \tilde{A}_{X}^{0, q}(\bar{V}) \rightarrow \tilde{A}_{X}^{1, q}(\bar{V})\right]=\bar{\Omega}^{q} \otimes j_{*} \tilde{\mathfrak{g}}, \quad \bigcirc
$$

In fact, when the set of eigenvalues of the monodromies around the irreducible components of the divisor $D$ does not include 1 , one can use cohomology with compact support to identify

$$
E_{1}^{p, q}=H_{c}^{p}\left(U, \bar{\Omega}^{q} \otimes \tilde{\mathfrak{g}}\right) .
$$

We define $\mathcal{L}^{p, q}(\tilde{\mathfrak{g}})_{(2)}$ to be the sheaf of measurable $(p, q)$-forms $\alpha$ on $U$ with values in $\tilde{\mathfrak{g}}$ such that both $\alpha$ and $\left(d^{\prime}+d^{\prime \prime}\right) \alpha$ are locally square-integrable. Here as usual we have the Poincaré metric near $D$ and the Kähler metric on $U$ which are compatible. It means that the Kähler metric near $x \in D$ has the same asymptotic form as the Poincaré metric on $\Delta \cap D$, where $\Delta$ is a small polycylinder centered at $x$ (cf. [8]). Set

$$
L^{p, q}(\tilde{\mathfrak{g}})_{(2)}:=\Gamma\left(X, \mathcal{L}^{p, q}(\tilde{\mathfrak{g}})_{(2)}\right) .
$$

We let

$$
H^{p, q}(U, \tilde{\mathfrak{g}})_{(2)}:=H^{q}\left(L^{p, \bullet}(\tilde{\mathfrak{g}})_{(2)}, d^{\prime \prime}\right) .
$$

Analogously one defines the space $H^{k}(U, \tilde{\mathfrak{g}})_{(2)}$. Let us recall the following result due to Timmerscheidt [7]:

PROPOSITION 2.4 (a)

$$
H^{k}(U, \tilde{\mathfrak{g}})_{(2)} \simeq \bigoplus_{p+q=k} H^{p, q}(U, \tilde{\mathfrak{g}})_{(2)}
$$

(b) The complexes of sheaves $\tilde{\Omega}_{X}^{p}(\bar{V})$ and $\mathcal{L}^{p, \bullet}(\tilde{\mathfrak{g}})_{(2)}$ are quasi-isomorphic, thus

$$
H^{q}\left(X, \tilde{\Omega}_{X}^{p}(\bar{V})\right) \simeq H^{p, q}(U, \tilde{\mathfrak{g}})_{(2)}
$$


(c) The spectral sequence

$$
E_{1}^{p, q}=H^{q}\left(X, \tilde{\Omega}_{X}^{p}(\bar{V})\right)
$$

degenerates at $E_{1}$, abuts to $H^{p+q}\left(X, j_{*} \tilde{\mathfrak{g}}\right)$ with

$$
H^{k}\left(X, j_{*} \tilde{\mathfrak{g}}\right) \simeq \bigoplus_{p+q=k} H^{q}\left(X, \tilde{\Omega}_{X}^{p}(\bar{V})\right)
$$

We have a double complex $\left(\tilde{A}_{X}^{p, q}(\bar{V}), d^{\prime}, d^{\prime \prime}\right)$ of differential forms on $X$ with coefficients in $\bar{V}$ with possible holomorphic logarithmic singularities along $D$ and without residues. The $d^{\prime \prime}$ cohomology of this double complex is $H^{q}\left(X, \tilde{\Omega}_{X}^{p}(\bar{V})\right)$ and the associated spectral sequence with

$$
E_{1}^{p, q}=H^{q}\left(X, \tilde{\Omega}_{X}^{p}(\bar{V})\right)
$$

degenerates at $E_{1}$ by the above Proposition. Therefore, we have the following consequence of our Theorem 2.3:

COROLLARY 2.5 There exists a spectral sequence with

$$
E_{1}^{p, q}=H^{p}\left(X, \bar{\Omega}^{q} \otimes j_{*} \tilde{\mathfrak{g}}\right)
$$

which abuts to $H^{p, q}(U, \tilde{\mathfrak{g}})_{(2)}$.

In fact, a simple computation shows that even for $X$ a Riemann surface of genus one with one puncture $\mathrm{P}$ and a local system of rank 1 on it with non-trivial monodromy around the puncture, the group

$$
H^{1}\left(X, \bar{\Omega}^{0} \otimes j_{*} \tilde{\mathfrak{g}}\right)=H_{c}^{1}\left(X \backslash P, \bar{\Omega}^{0} \otimes \tilde{\mathfrak{g}}\right)
$$

by Serre duality is dual to the space of the sections over $U$ of the sheaf $\bar{\Omega}^{1} \otimes \tilde{\mathfrak{g}}^{\prime}$. Here $\tilde{\mathfrak{g}}^{\prime}$ stands for a local system dual to $\tilde{\mathfrak{g}}$. It means that in general the spaces $E_{1}^{p, q}$ are infinite-dimensional. Thus the spectral sequence that we constructed does not degenerate at $E_{1}$ unless the local system extends to $X$. We apply Proposition 5.17 from [3] to see that in this case the DGLA $\left(\tilde{A}_{X}^{\bullet}(\bar{V}), d^{\prime}+d^{\prime \prime}\right)$ given by

$$
\tilde{A}_{X}^{i}(\bar{V})=\bigoplus_{p+q=i} \tilde{A}_{X}^{p, q}(\bar{V})
$$


does not satisfy the $d^{\prime} d^{\prime \prime}$-Lemma.

In fact, in [4] the author constructs another DGLA $B^{\bullet}(\tilde{\mathfrak{g}})$, for which the $d^{\prime} d^{\prime \prime}$-Lemma holds implying its formality, which is important for the purposes of deformation theory. Moreover, there is a natural DGLA inclusion

$$
\tilde{A}_{X}^{\bullet}(\bar{V}) \hookrightarrow B^{\bullet}(\tilde{\mathfrak{g}}),
$$

which induces an isomorphism on cohomology. This means that the DGLA $\tilde{A}_{X}^{\bullet}(\bar{V})$ that we consider here is formal too despite the failure of the $d^{\prime} d^{\prime \prime}$ Lemma.

\section{References}

[1] P. Deligne, Equations Différentielles a Points Singuliers Réguliers, Lect. Notes in Math. 163, Springer-Verlag, 1970

[2] P. Deligne, Théorie de Hodge II, Publ. Math. IHES, 40, 1971, 5-58

[3] P. Deligne, Ph. Griffiths, J. Morgan and D. Sullivan, Real homotopy theory of Kähler manifolds, Invent. Math., 29, 1975, 245-274

[4] P. A. Foth, Deformations of representations of fundamental groups of open Kähler manifolds, preprint, dg-ga/9709013

[5] R. Gunning, Introduction to holomorphic functions of several variables, Vol. I, II, III, Wadsworth $\& 3$ Brooks/Cole, 1990

[6] H. Hironaka, Resolution of singularities of an algebraic variety over a field of characteristic zero I, II, Ann. of Math., 79, 1964, no. 1 \& 2

[7] K. Timmerscheidt, Hodge decomposition for unitary local sysems, Appendix to: H. Esnault, E. Viehweg, Logarithmic de Rham complexes and vanishing theorems, Invent. Math., 86, 1986, 161-194

[8] S. Zucker, Hodge theory with degenerating coefficients: $L_{2}$-cohomology in the Poincaré metric, Ann. Math., 109, 1979, 415-476

Department of Mathematics Penn State University 
University Park, PA 16802

foth@math.psu.edu

AMS subj. class.: 17B70, 14F05. 